OPEN ACCESS

Edited by:

Yun Qian,

Shanghai Jiao Tong University, China

Reviewed by:

Yanbin Kuang,

Shanghai JiaoTong University, China

Shuyuan Wang,

Shanghai Jiaotong University, China

${ }^{*}$ Correspondence:

Lin Zhou

Izhou@cdc.zj.cn

Guanyang Zou

zgy1021@hotmail.com

Specialty section:

This article was submitted to Infectious Diseases - Surveillance,

Prevention and Treatment,

a section of the journal

Frontiers in Public Health

Received: 13 August 2021

Accepted: 04 October 2021

Published: 18 November 2021

Citation:

Xiao W, Chen B, Huang D, Chan O,

Wei $X$, Zhou L and Zou G (2021)

Comparison of Delay in Tuberculosis

Diagnosis Between Migrants and

Local Residents in an Eastern County

of China: An Analysis of the Electronic

Data Between 2015 and 2019.

Front. Public Health 9:758335.

doi: 10.3389/fpubh.2021.758335

\section{Comparison of Delay in Tuberculosis Diagnosis Between Migrants and Local Residents in an Eastern County of China: An Analysis of the Electronic Data Between 2015 and 2019}

\author{
Wenhui Xiao ${ }^{1}$, Bin Chen ${ }^{2}$, Dajiang Huang ${ }^{3}$, Olivia Chan ${ }^{4}$, Xiaolin Wei ${ }^{5}$, Lin Zhou ${ }^{2 *}$ and \\ Guanyang Zou ${ }^{1 *}$
}

\begin{abstract}
${ }^{1}$ School of Public Health and Management, Guangzhou University of Chinese Medicine, Guangzhou, China, ${ }^{2}$ Zhejiang Provincial Center for Disease Control and Prevention, Hangzhou, China, ${ }^{3}$ Center for Disease Control and Prevention, Cangnan County, Wenzhou, China, ${ }^{4}$ School of Public Health, University of Hong Kong, Hong Kong SAR, China, ${ }^{5}$ Division of Clinical Epidemiology \& Institute of Health Policy, Management and Evaluation, Dalla Lana School of Public Health, University of Toronto, Toronto, ON, Canada
\end{abstract}

Introduction: China continues to rank among one of the countries with the highest number of tuberculosis (TB) cases globally. Migrants are a particularly at-risk subgroup for TB and pose a challenge for case management in contemporary China. The early diagnosis and treatment of patients with TB are pivotal for effective TB control. This study investigates the delay in the TB diagnosis of migrants as compared with residents, to provide an evidence base for improved case detection and the better management of migrant patients with TB.

Materials and Methods: The data was collected from the Tuberculosis Information Management System (TBIMS) (2015-2019) in an eastern county of China. The total diagnostic delay, consisting of patient delay and health system delay, is defined as the interval between the onset of TB symptoms and the confirmation of TB diagnosis in the designated TB hospital. The comparison of the delay in the TB diagnosis between migrants and residents was conducted using a Mann-Whitney $U$-test and chi-square test. The difference in the delay curves between these two groups was examined using a log-rank test.

Results: Of 2,487 patients with TB, 539 (22\%) were migrants. The migrants tended to be younger, presented with less severe conditions, received an initial diagnosis at prefectural and above-level hospitals. Compared with the local patients with TB, the migrant patients with TB had a longer median total diagnostic delay (30 vs. 9, $P=0.000$ ) and a higher proportion of patients with this delay $>28$ days (52 vs. $13 \%, P=0.000$ ). Similarly, the migrant patients with TB also had a longer median patient delay (13 vs. $9, P=0.000$ ) and a higher proportion of patients with this delay $>14$ days (47 vs. $30 \%, P=0.000$ ), longer median health system delay ( 9 vs. $0, P=0.000$ ), and a higher proportion of patients with 
this delay $>14$ days (42 vs. $0.5 \%, P=0.000$ ) than the local patients with TB. The survival curves of delay showed that the longer the time interval was, the more likely the migrant patients with TB were to be diagnosed $(P<0.05)$.

Conclusions: Diagnosis is significantly delayed among migrant patients with TB. Our study highlights the importance of early screening and diagnosis for TB especially among migrants, to improve access and ensure better management for all patients with TB.

Keywords: migrants, tuberculosis, total diagnostic delay, patient delay, health system delay

\section{INTRODUCTION}

Tuberculosis is a communicable disease, the leading cause of death ranking above HIV/AIDS. Globally, an estimate of 10 million people fell ill with tuberculosis (TB), and 1.4 million people died from this disease in 2019 (1). It is generally believed that TB is a disease of poverty, as $98 \%$ of TB cases were reported from 121 low-and middle-income countries in the same year (1). China is listed as one of the $30 \mathrm{~TB}$ high burden countries in the world. In 2019, an estimated 8,33,000 people fell ill with TB in China, accounting for $8.4 \%$ of the global TB cases burden (1). Over the past two decades, there has been significant progress in TB control in China, with the incidence being halved and mortality reduced by more than $70 \%$ (1). Nevertheless, China is still facing tremendous challenges in TB control, including a high TB burden and difficulties in case detection and management among migrants.

In China, the migrant populations are mostly internal migrants (henceforth called migrants). In 2019, the number of migrants in China reached 236 million, accounting for onesixth of the whole population (2). Most migrants in China have traveled from rural areas to prosperous economic regions to earn higher incomes and find better opportunities $(3,4)$. The large population of migrants and domestic flows have had considerable impacts on the TB epidemic in China. A national study reported that migrants accounted for one-fifth of all the TB cases nationwide between 2014 and 2015, representing 0.3 million migrant patients with TB (5). Migrants are always considered as an important vulnerable subgroup among patients with TB (6). Compared with residents, migrants face substantial problems in accessing medical care, insurance, and social security (7). Many studies reported poor health-service-seeking behavior among migrants in China (8-11), and these studies indicated the inequities of accessing health services among migrants and residents.

The early detection and timely treatment of migrant patients with TB are essential for effective TB control and are emphasized in the "End TB strategy" of the WHO $(6,12)$. Delays occurring during the process of diagnosis and treatment increase the probability of TB transmission and eventually result in a higher disease burden (13). In most places in China, patients with TB

Abbreviations: TB, Tuberculosis; WHO, World Health Organization; TBIMS, China's Tuberculosis information management system; GDP, Gross Domestic Products; CDCs, Center for Disease Prevention and Control; IQR, Interquartile Range. receive standardized diagnosis and treatment in the designated general hospital of the county, and other health facilities refer patients with $\mathrm{TB}$ or presumptive patients with $\mathrm{TB}$ to the designated TB hospitals for standardized diagnosis and treatment (14). Numerous studies have focused on the delay in TB diagnosis for migrants in China $(13,15-20)$. However, most of these studies in China were conducted in big cities such as Shanghai and Shenzhen. Few have studied the delay in TB diagnosis for migrants living in the counties. In a cross-sectional study conducted in 12 counties of Shandong Province, Zhou et al. reported a median total diagnostic delay, patient delay, and health system delay of 21,10 , and 8 days among migrant patients with TB (13). They also found that a proportion of 41 and $45 \%$ migrant patients with TB have patient delay and health system delay of $>14$ days (13). Also, current evidence regarding the probability of being diagnosed at different time intervals for patients with TB (including migrants and residents) is limited. Chen et al. assessed the probability of being diagnosed at different months since the onset of TB symptoms for patients with TB to understand the delay of diagnosis using survival analysis (21). This study analyzes the TB diagnosis delay among the migrant patients with TB as compared with local patients with TB registered between 2015 and 2019 in Cangnan County, Zhejiang province, to provide an evidence base for the improved case detection and better management of migrant patients with TB.

\section{MATERIALS AND METHODS}

\section{Study Design}

This is a retrospective study of the diagnostic delay of migrant patients with TB as compared with local patients with TB over 5 years (2015-2019). The data was collected from the patient records exported from the Tuberculosis Information Management System of China (TBIMS) (TB special reporting system version 2.0).

\section{Study Setting}

This study was conducted in Cangnan County of Wenzhou City, Zhejiang Province. Zhejiang is one of the most developed provinces in China and a key destination for migrants. Wenzhou is a city under the jurisdiction of Zhejiang Province. In 2019, Wenzhou had a total gross domestic product (GDP) exceeding the US $\$ 100$ billion, ranking third out of 11 cities in Zhejiang province, with an average per capita GDP of $\$ 10,000$ (22). Wenzhou City consists of four districts, five counties, and three county-level cities with a population of 9.3 million (22). Cangnan 
is a populous and well-off county in Wenzhou, with a population of 1.24 million and an average per capita GDP of $\$ 9,200$ (22). In each county of Wenzhou City, there is a designated general hospital responsible for $\mathrm{TB}$ diagnosis and treatment, and in Cangnan, it is the Cangnan County People's Hospital.

\section{Data Collection}

This study used the routine electronic practice data that were collected and recorded by $\mathrm{TB}$ health providers during $\mathrm{TB}$ consultation at the time of $\mathrm{TB}$ registration. This study selected the data of patients with TB registered from January 1, 2015, to December 31, 2019. The data was exported from TBIMS to Microsoft Excel (Microsoft, Redmond, Washington, United States) by the staff in the Center for Disease Control and Prevention (CDC) of Zhejiang Province and Cangnan. The data covers the demographics of patients with TB such as age, sex, occupation, patient source, level of the hospital for initial TB diagnosis, and household registration (migrant or local resident); clinical characteristics such as smear sputum results, cavity, TB severity (e.g., with large cavities or lesions in more than two lung lobes), treatment category (new or retreated cases), treatment outcomes; and health service-related information such as date of onset of TB symptoms, date of first health-care visit, and date of confirmed TB diagnosis.

\section{Definitions}

In this study, patients with $\mathrm{TB}$ who did not have household registration in Cangnan County or who lived in Cangnan County for $<6$ months at the time of registration was classified as "migrant" in the TBIMS. In this study, we analyzed the total diagnostic delay, patient delay, and health system delay of migrant patients with $\mathrm{TB}$ as compared with local patients with TB. The total diagnostic delay (henceforth called total delay) is the sum of the patient delay and health system delay. The patient delay and health system delay is defined as the interval between the onset of TB symptoms and the first visit to a health facility, and the interval between the first visit to a health facility and confirmed TB diagnosis in the TB designated hospital, respectively (23). We used 28 days as a cut-off point for the analysis of total delay and 14 days as a cut-off point for the analysis of patient delay and health system delay based on previous studies (24-26). Tuberculosis diagnosis is mainly based on sputum smear examination, supplemented by sputum culture and X-Ray. To diagnose TB, suspected patients with TB need to have three samples of smear sputum to be collected and tested, i.e., a sample of "instant sputum" on the same day of TB clinic visit, a second sample of "night sputum" and a third sample of “morning sputum" next day (27).

\section{Data Analysis}

The data were analyzed using SPSS 21.0 (SPSS, Inc., Chicago, United States). The demographics, clinical symptoms, and delay in TB diagnosis for the migrant and local patients with TB were depicted using descriptive statistics. The continuous variables were presented by the median and interquartile range (IQR), while the categorical variables were presented by counts and proportions. The delay in the TB diagnosis (including delay times and delay proportion) between the migrant and local patients with TB was compared using a Mann-Whitney $U$ test and Chi-square test. In addition, we estimated the probability of the migrant patients with $\mathrm{TB}$ being diagnosed at different time intervals (e.g., $1^{*} 14$ days) based on three diagnostic delay categories, as compared with the local residents, further testifying and comparing the diagnostic delay among these two cohorts. Hence, Kaplan-Meier survival curves were drawn to record the median times of total delay, patient delay, and health system across 5 years. The difference in these delay curves between migrant and local patients with TB was examined using a Log-rank test. A $p$-value of $<0.05$ was considered statistically significant.

\section{RESULTS}

\section{Demographics and Clinic Symptoms of Migrant TB Patients as Compared With Local TB Patients}

A total of 2,487 TB cases were reported between 2015 and 2019 in Cangnan County, Zhejiang Province, including 539 migrants $(22 \%)$ and 1,948 residents (78\%). The migrant patients with TB were significantly younger than the local patients with TB (39 vs. 49 years, $p<0.05)$ and had a lower proportion of patients who were farmers ( 39 vs. $57 \%, p<0.05$ ), with a cavity ( 33 vs. $39 \%$, $p$ $<0.05$ ), and severe cases (27 vs. $38 \%, p<0.05$ ). Compared with the local patients with TB, the migrant patients with TB had a lower proportion of patients who were referred to TB designated hospitals from other health facilities ( 35 vs. $77 \%, p<0.05$ ), and firstly diagnosed at county-level TB designated hospitals (35 vs. $99 \%, p<0.05)$ over 5 years. In terms of treatment outcomes, the migrant patients with TB were more likely to end up with unfavorable outcomes than the local patients with TB (15 vs. 4\%, $p<0.05)$. For instance, the proportion of the migrant patients who were unable to follow-up during the treatment was $7 \%$, as compared with $0.6 \%$ of the local patients (Table $\mathbf{1}$ ).

\section{Total Delay, Patient Delay, and Health System Delay of Migrant TB Patients as Compared With Local TB Patients}

The median (IQR) total delay for the migrant patients with TB was 30 (11-64) days, significantly longer than the 9 (4-17) days for the local patients with TB over 5 years $(P<0.05)$. In addition, compared with the local patients with $\mathrm{TB}$, the migrant patients with TB experienced significantly longer total delays every year from 2015 to $2019(P<0.05)$. The proportion with a total delay of $>28$ days for the migrant patients with TB was $52 \%$, significantly higher than the $13 \%$ for the local patients with TB over 5 years $(P<0.05)$. In addition, compared with the local patients with TB, the migrant patients with TB had a significantly higher proportion of patients whose total delay was $>28$ days every year from 2015 to 2019 ( $P<0.05$ ) (Table 2).

The median (IQR) patient delay for the migrant patients with TB was 13 (4-34) days, significantly longer than the 9 (4-17) days for local patients with TB over 5 years $(P<0.05)$. In addition, compared with the local patients with $\mathrm{TB}$, the migrant patients 
TABLE 1 | Demographics, clinical characteristics of migrant and local patients with TB from 2015 to 2019.

\begin{tabular}{|c|c|c|c|c|c|c|c|c|c|c|c|c|c|}
\hline & \multirow[b]{2}{*}{2015} & \multicolumn{5}{|c|}{ Migrant TB patients $(n=539)$} & \multicolumn{6}{|c|}{ Local TB patients $(n=1948)$} & \multirow[b]{2}{*}{$P$-value } \\
\hline & & 2016 & 2017 & 2018 & 2019 & Total & 2015 & 2016 & 2017 & 2018 & 2019 & Total & \\
\hline Age (Median, IQR) & $29(22-49)$ & $36(25-52)$ & $44(31-56)$ & $42(26-54)$ & $41(28-54)$ & $39(26-53)$ & 49 33-61) & $46(30-60)$ & $51(37-65)$ & $49(31-63)$ & $52(35-65)$ & $49(33-63)$ & 0.000 \\
\hline Age $>45$ & $26(29)$ & $40(40)$ & $45(44)$ & $47(40)$ & $53(41)$ & $211(39)$ & $265(55)$ & $206(51)$ & $246(64)$ & $203(56)$ & $194(61)$ & $1,114(57)$ & 0.000 \\
\hline Male & $61(69)$ & $71(70)$ & $69(68)$ & $85(73)$ & $94(72)$ & $380(71)$ & $349(73)$ & $295(73)$ & $287(74)$ & $259(72)$ & $242(76)$ & $1,432(74)$ & 0.164 \\
\hline Farmer & $43(48)$ & $48(48)$ & $34(33)$ & $27(23)$ & $38(30)$ & $190(35)$ & $432(90)$ & $340(85)$ & $309(80)$ & $207(57)$ & $217(69)$ & $1,505(77)$ & 0.000 \\
\hline Smear positive & $40(45)$ & $32(32)$ & $41(40)$ & $35(30)$ & $35(27)$ & $183(34)$ & $174(36)$ & $113(28)$ & $136(35)$ & $146(40)$ & $114(36)$ & $683(35)$ & 0.632 \\
\hline Cavity & $39(44)$ & $37(37)$ & $37(36)$ & $31(27)$ & $34(27)$ & $178(33)$ & $182(38)$ & $153(38)$ & $156(40)$ & $136(38)$ & $134(42)$ & 761 (39) & 0.012 \\
\hline Severe cases & $38(43)$ & $33(33)$ & $33(32)$ & $17(15)$ & $22(17)$ & $143(27)$ & $172(36)$ & $150(37)$ & $155(40)$ & $127(35)$ & $130(41)$ & $734(38)$ & 0.000 \\
\hline Patient source ${ }^{a}$ & & & & & & & & & & & & & 0.000 \\
\hline Symptomatic visits & $44(49)$ & $77(76)$ & $73(72)$ & $80(70)$ & $39(30)$ & $313(68)$ & $111(23)$ & $97(24)$ & $60(16)$ & $32(9)$ & $25(8)$ & $325(17)$ & \\
\hline Referral & $41(46)$ & $23(23)$ & $24(24)$ & $22(19)$ & $34(26)$ & 144 (32) & $368(77)$ & $305(76)$ & $323(84)$ & 329 (91) & 291 (92) & $1,616(83)$ & \\
\hline Treatment category & & & & & & & & & & & & & 0.945 \\
\hline New & $76(85)$ & $80(79)$ & $90(88)$ & $108(92)$ & $118(91)$ & $472(88)$ & $425(89)$ & $353(88)$ & $332(86)$ & $311(86)$ & $287(91)$ & $1,708(88)$ & \\
\hline Retreated & $13(15)$ & $21(21)$ & $12(12)$ & $9(8)$ & $12(9)$ & $67(12)$ & $55(11)$ & $49(12)$ & $55(14)$ & $51(14)$ & $30(9)$ & 240 (12) & \\
\hline \multicolumn{14}{|c|}{ Level of hospital for initial TB diagnosis } \\
\hline County & $48(54)$ & $36(36)$ & 36 (35) & $29(25)$ & $38(29)$ & 187 (35) & 479 (99) & 400 (99) & 386 (99) & 361 (99) & 315 (99) & $1,941(99)$ & 0.000 \\
\hline Prefectural and above & $41(46)$ & $65(64)$ & $66(65)$ & $88(75)$ & $92(71)$ & $352(65)$ & $1(1)$ & $2(1)$ & $1(1)$ & $1(1)$ & $2(1)$ & $7(1)$ & \\
\hline Treatment outcomes $^{b}$ & & & & & & & & & & & & & 0.000 \\
\hline Treatment success & $80(90)$ & $76(75)$ & $88(86)$ & $99(90)$ & $7(70)$ & 350 (85) & 470 (98) & 395 (98) & 367 (95) & 331 (95) & $13(62)$ & $1,576(96)$ & \\
\hline Cured & $36(40)$ & $20(20)$ & $37(36)$ & $29(26)$ & $1(10)$ & $123(30)$ & 167 (35) & $110(27)$ & $122(32)$ & $132(38)$ & $1(5.0)$ & 532 (32) & \\
\hline Treatment completed & $44(49)$ & $56(55)$ & $51(50)$ & $70(64)$ & $6(60)$ & 227 (55) & 303 (63) & $285(71)$ & $245(63)$ & $199(57)$ & $12(57)$ & $1,044(64)$ & \\
\hline Unfavorable outcomes & $9(10)$ & $25(25)$ & $14(14)$ & $11(10)$ & $3(30)$ & $62(15)$ & $10(2.0)$ & $7(2.0)$ & $20(5.0)$ & $17(5.0)$ & $8(38)$ & $62(4.0)$ & \\
\hline Lost to follow-up & $5(5.6)$ & $12(12)$ & $8(7.8)$ & $4(3.6)$ & $0(0.0)$ & $29(7.0)$ & $0(0.0)$ & $0(0.0)$ & $7(1.8)$ & $3(0.9)$ & $0(0.0)$ & $10(0.6)$ & \\
\hline Treatment failed & $1(1.1)$ & $2(2.0)$ & $1(1.0)$ & $0(0.0)$ & $0(0.0)$ & $4(1.0)$ & $6(1.3)$ & $1(0.2)$ & $6(1.6)$ & $4(1.1)$ & $3(14)$ & $20(1.2)$ & \\
\hline Diagnostic change & $0(0.0)$ & $5(5.0)$ & $3(2.9)$ & $4(3.6)$ & $1(10)$ & $13(3.2)$ & $2(0.4)$ & $3(0.7)$ & $2(0.5)$ & $7(2.0)$ & $2(9.5)$ & $16(1.0)$ & \\
\hline Transfer to MDR & $0(0.0)$ & $2(2.0)$ & $1(1.0)$ & $2(1.8)$ & $1(10)$ & $6(1.5)$ & $1(0.2)$ & $1(0.2)$ & $0(0.0)$ & $2(0.6)$ & $1(5.0)$ & $5(0.3)$ & \\
\hline Adverse reactions & $0(0.0)$ & $0(0.0)$ & $0(0.0)$ & $1(0.9)$ & $0(0.0)$ & $1(0.2)$ & $0(0.0)$ & $0(0.0)$ & $0(0.0)$ & $0(0.0)$ & $0(0.0)$ & $0(0.0)$ & \\
\hline Died & $1(1.1)$ & $1(1.0)$ & $0(0.0)$ & $0(0.0)$ & $1(10)$ & $3(0.7)$ & $1(0.2)$ & $2(0.5)$ & $4(1.0)$ & $0(0.0)$ & $0(0.0)$ & $7(0.4)$ & \\
\hline Other & $2(2.2)$ & $3(3.0)$ & $1(1.0)$ & $0(0.0)$ & $0(0.0)$ & $6(1.5)$ & $0(0.0)$ & $0(0.0)$ & $1(0.3)$ & $1(0.3)$ & $2(9.5)$ & $4(0.2)$ & \\
\hline
\end{tabular}

Unless indicated otherwise, data are given as $n(\%)$.

a In addition to symptomatic visits and referral, there were also 43\% migrant patients with TB that came from close contact tracing in 2019.

$\mathrm{b}$ In this variable, we presented treatment outcomes of 2050 patients who stopped treatment before the data was exported from TBIMS 
TABLE 2 | The total delay of migrant and local patients with TB from 2015 to 2019.

\begin{tabular}{|c|c|c|c|c|c|c|}
\hline \multirow[t]{2}{*}{ Year } & \multicolumn{3}{|c|}{ Days (Median, IQR) } & \multicolumn{3}{|c|}{ Proportion ( $n, \%)$} \\
\hline & Migrants & Local & $P$-value & Migrants & Local & $P$-value \\
\hline 2015 & $29(10-52)$ & $9(4-19)$ & 0.000 & $45(51)$ & $63(13)$ & 0.000 \\
\hline 2016 & $30(12-60)$ & $8(4-16)$ & 0.000 & $53(53)$ & $47(12)$ & 0.000 \\
\hline 2017 & $38(16-78)$ & $10(4-21)$ & 0.000 & $61(60)$ & $63(16)$ & 0.000 \\
\hline 2018 & $49(21-105)$ & $8(3-18)$ & 0.000 & $83(71)$ & $53(15)$ & 0.000 \\
\hline 2019 & $17(7-33)$ & $9(3-14)$ & 0.000 & $38(29)$ & $31(10)$ & 0.000 \\
\hline 2015-2019 & $30(11-64)$ & $9(4-17)$ & 0.000 & $280(52)$ & $257(13)$ & 0.000 \\
\hline
\end{tabular}

with TB experienced significantly longer patient delays every year from 2015 to 2018 ( $P<0.05)$. However, the migrant patients with TB experienced shorter patient delays as compared with the local patients with TB in 2019 ( 4 vs. 9 days, $p<0.05$ ). The proportion with a patient delay of $>14$ days for the migrant patients with TB was $47 \%$, significantly higher than the $30 \%$ for the local patients with TB over 5 years $(P<0.05)$. In addition, compared with the local patients with $\mathrm{TB}$, the migrant patients with $\mathrm{TB}$ had a significantly higher proportion of patients whose patient delay was $>14$ days every year from 2015 to $2018(P<0.05)$. However, no significant difference was observed among these two groups regarding patient delay $>14$ days in $2019(P>0.05)$ (Table 3).

The median (IQR) health system delay for the migrant patients with TB was $9(0-25)$ days, which is significantly longer than the $0(0-0)$ days for the local patients with TB over 5 years $(P<0.05)$. In addition, compared with the local patients with $\mathrm{TB}$, the migrant patients with TB experienced significantly longer health system delays every year from 2015 to $2019(P<0.05)$. The proportion with a health system delay of $>14$ days for the migrant patients with $\mathrm{TB}$ was $42 \%$, significantly higher than the $0.5 \%$ for the local patients with TB over 5 years $(P<0.05)$. In addition, compared with the local patients with TB, the migrant patients with TB had a significantly higher proportion of patients whose patient delay was >14 days every year from 2015 to 2019 too $(P<0.05)$ (Table 4$)$.

\section{Survival Curves}

The log-rank test showed that the total delay curves significantly differed between the migrant and local patients with TB over 5 years (Log-rank test $\chi^{2}=268.409, p<0.001$ ). The probability of the migrant patients with TB being diagnosed within 28 days since the onset of symptoms was $48 \%$ as compared with the $87 \%$ of local patients with TB. Furthermore, it took up to $8^{*} 28$ days for $95 \%$ of the migrant patients with $\mathrm{TB}$ to be diagnosed since the onset of TB symptoms, and $2 * 28$ days for $95 \%$ of the local patients with TB to be diagnosed since the onset of TB symptoms (Figure 1).

The log-rank test showed that the patient delay curves significantly differed between the migrant and local patients with TB over 5 years (Log-rank test $\chi^{2}=78.135, p<0.001$ ). The probability of the migrant patients with $\mathrm{TB}$ visiting health facilities within 14 days of onset TB symptoms was 53\% as compared with the $70 \%$ of the local patients with TB. Furthermore, it took up to $12 * 14$ days and longer for $95 \%$ of the migrant patients with TB to visit health facilities since the onset of TB symptoms, and $4^{*} 14$ days for $95 \%$ of the local patients with TB to visit health facilities since the onset of TB symptoms (Figure 2).

The log-rank test showed that the health system delay curves significantly differed between the migrants and local patients with TB over 5 years (Log-rank test $\left.\chi^{2}=1,169.030, p<0.001\right)$. The probability of the migrant patients with TB getting a confirmed diagnosis within 14 days after the first visit to a health facility was $58 \%$ as compared with the $99.5 \%$ of the local patients with TB. Furthermore, it took up to $5^{*} 14$ days and longer for $95 \%$ of the migrant patients with TB to get a confirmed diagnosis after their first visit to a health facility. However, the health system delay curve for the local patients with TB was the infinitely approaching point " 1 " from the beginning, which showed that the local patients with TB have nearly $100 \%$ probability of getting a confirmed diagnosis on the same day when they first visited a health facility (Figure 3).

\section{DISCUSSION}

\section{Summary of Findings}

In this study, we found that migrant patients with TB tended to be younger, non-farmer, have less severe conditions, receive an initial diagnosis at prefectural and above-level hospitals. The median total delay, patient delay, and health system delay for migrant patients with TB were 30,13, and 9 days, respectively, as compared with 9,9 , and 0 days for local patients with TB. Compared with local patients with $\mathrm{TB}$, migrant patients with $\mathrm{TB}$ had a higher proportion of patients with a total delay of $>28$ days, patient delay of $>14$ days, and health system delay of $>14$ days. The survival curves of delay showed that the longer the time interval was, the more likely the migrant patients with TB were to be diagnosed.

\section{Comparison With Literature}

Migrants remain one of the most important vulnerable subgroups in the context of the TB epidemic. Tuberculosis control in migrants is pivotal for countries to progress toward TB elimination in accordance with the WHO END TB strategy (12). In this study, we found that migrants accounted for $22 \%$ of all the registered TB cases between 2015 and 2019 in a populous County of an Eastern Province in China. This was slightly higher than the $18 \%$ reported by $\mathrm{Li}$ et al. in a national study during 
TABLE 3 | Patient delay of migrant and local patients with TB from 2015 to 2019.

\begin{tabular}{|c|c|c|c|c|c|c|}
\hline Year & \multicolumn{3}{|c|}{ Days (Median, IQR) } & \multicolumn{3}{|c|}{ Proportion (n, \%) } \\
\hline 2016 & 17 (6-35) & $8(4-16)$ & 0.000 & 54 (53) & $112(28)$ & 0.000 \\
\hline 2017 & $20(6-53)$ & $10(4-21)$ & 0.000 & $55(54)$ & $143(37)$ & 0.002 \\
\hline 2018 & $28(8-59)$ & $8(3-17)$ & 0.000 & $68(58)$ & $106(29)$ & 0.000 \\
\hline 2015-2019 & 13(4-34) & $9(4-17)$ & 0.000 & $251(47)$ & $587(30)$ & 0.000 \\
\hline
\end{tabular}

TABLE 4 | Health system delay of migrant and local patients with TB from 2015 to 2019.

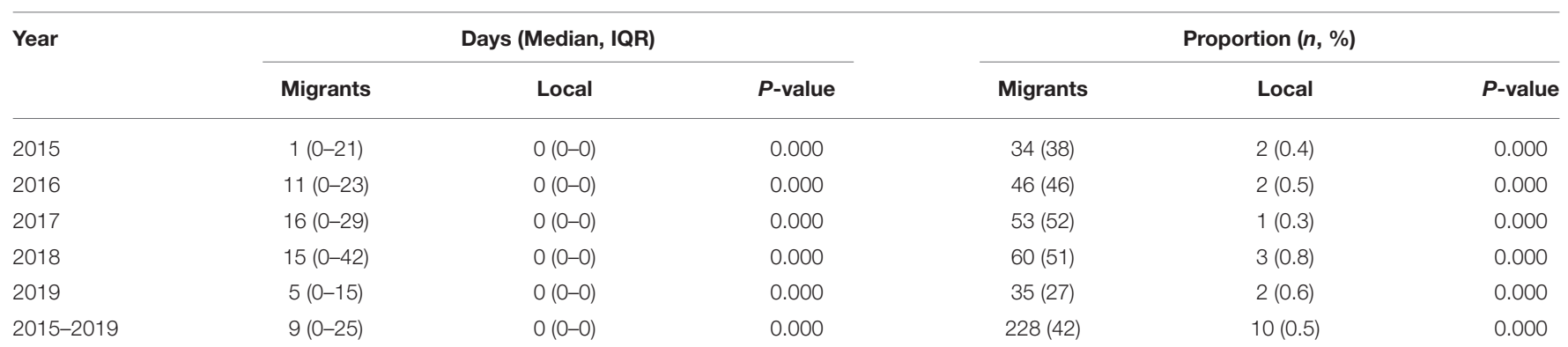

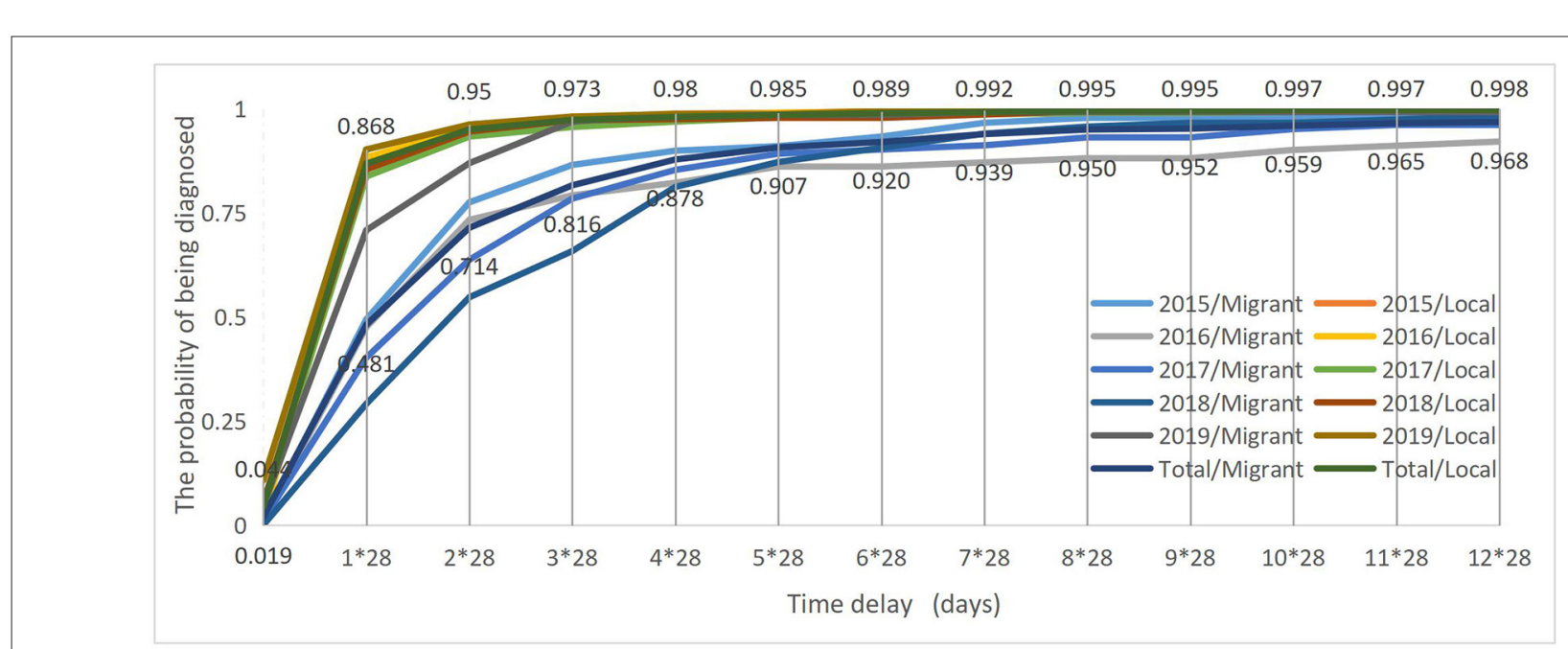

FIGURE 1 | Total delay curves between migrant and local patients with TB from 2015 to 2019.

2014-2015 (5), yet was much lower than the $72 \%$ in the study of Zhao conducted in Shanghai, a metropolis experiencing rapid economic development where the majority of the population are migrants (19).

Similar to the previous studies $(8,28-30)$, the migrants in our studies were younger and presented with less severe clinical symptoms as compared with the residents. This phenomenon is associated with the "healthy migrant effect" and reflects the self-selection of younger and healthier individuals to pursue higher incomes and better opportunities in more economically developed areas. In China, the tendency for people to seek health care at higher-level hospitals is universal (31). Our findings substantiate the phenomenon as more migrants seek medical care in tertiary hospitals compared with residents. One possibility is the geographic proximity of migrants to the County Center, with easier access to tertiary hospitals in the city. Similar to previous studies (32), we found that migrant patients with TB had poorer treatment outcomes, especially with a higher lostto-follow-up rate than local patients. This is mainly due to the "floating" characteristics of migrants and the lack of health insurance among the migrants in the host cities (33).

The main purpose of this study is to examine the delayed diagnosis of migrant patients with $\mathrm{TB}$ as compared with local patients with TB. In this study, we reported a median total delay 


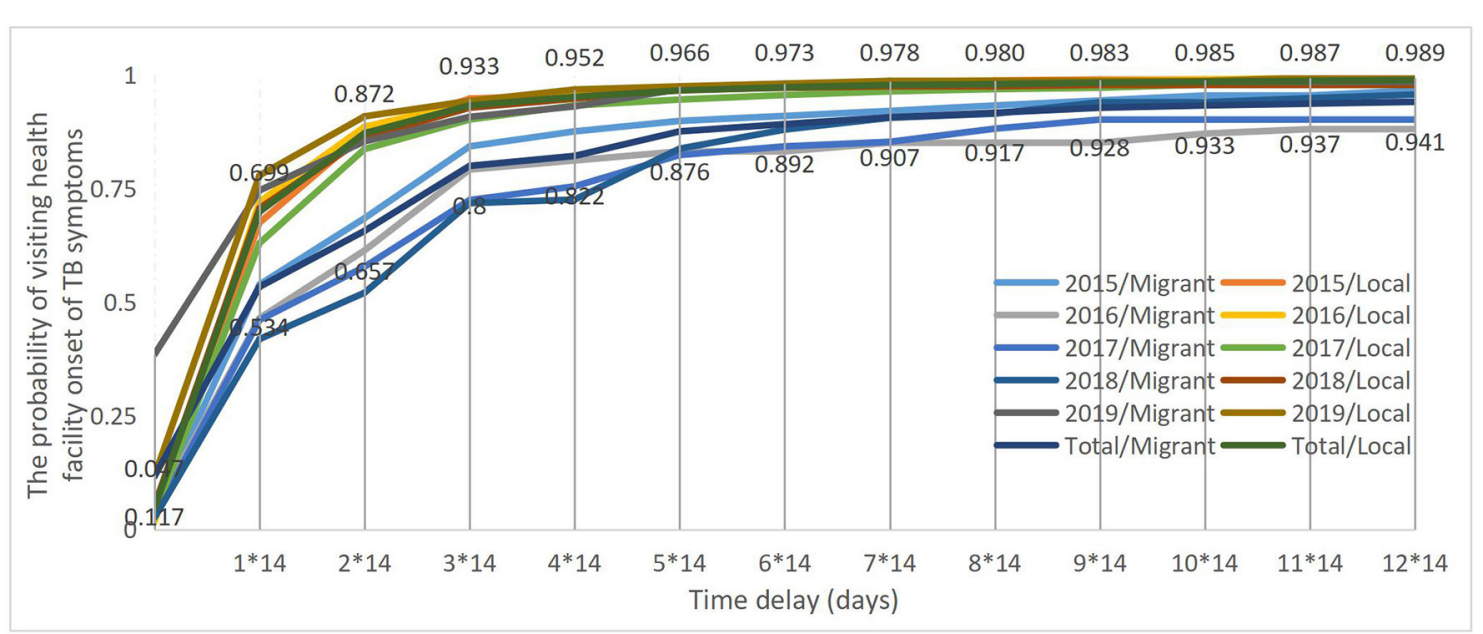

FIGURE 2 | Patient delay curves between migrant and local patients with TB from 2015 to 2019.

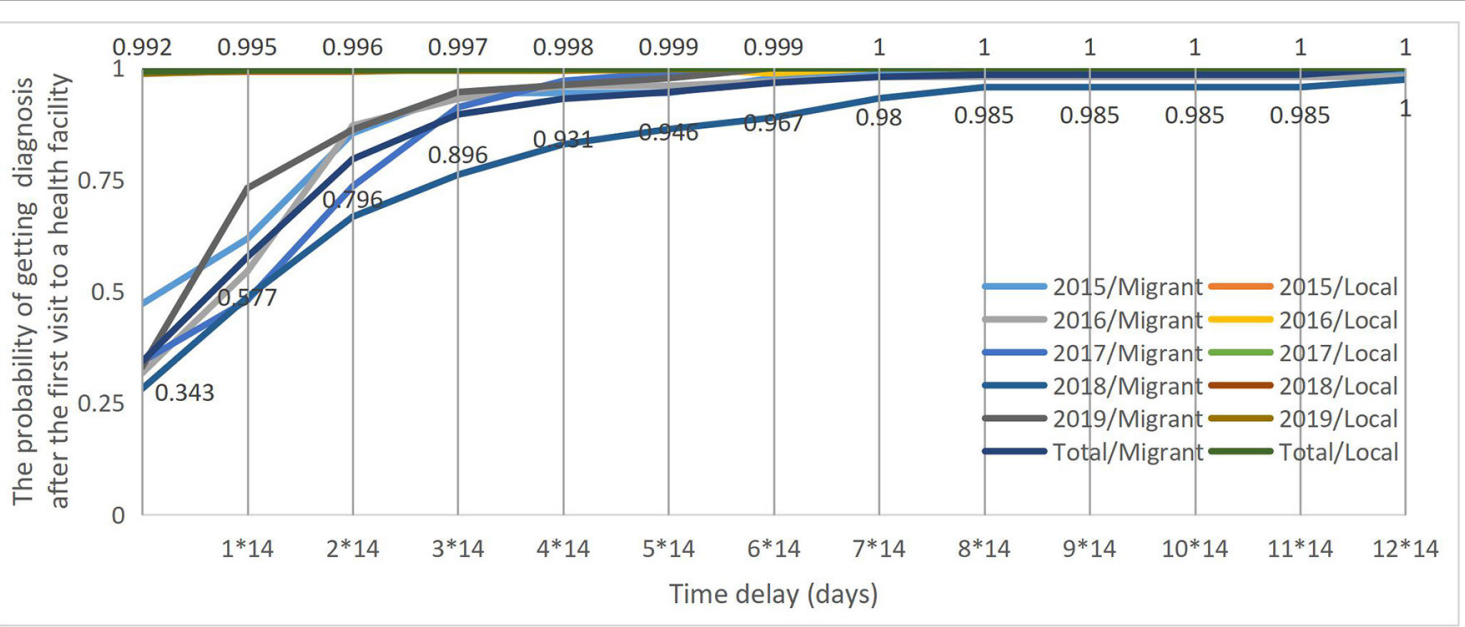

FIGURE 3 | Health system delay curves between migrant and local patients with TB from 2015 to 2019.

of 30 days for the migrant patients with TB, which is between the 21 days reported by Zhou et al. in the counties of Shandong and 46 days reported by Xiao et al. in the counties of Zhejiang $(13,23)$. Our study found that migrant patients with TB had a longer total delay as compared with local patients with TB, which is similar to an earlier study focused on patients with $\mathrm{TB}$ with diabetes mellitus (DM) comorbidity (23). We also found that migrant patients with $\mathrm{TB}$ had a higher proportion of patients with a total delay of $>28$ days.

Our study reported a median patient delay of 13 days and a proportion of $47 \%$ with patient delay $>14$ days for migrant patients with TB. Previous studies have reported a median patient delay ranging from 10 to 21 days and a proportion ranging from 39 to $68 \%$ with patient delay $>14$ days for migrant patients with TB in China $(13,15,16,18,19,23,34)$. The present study found that migrant patients with TB had a longer patient delay as compared with local patients with TB. However, two studies conducted in Shanghai reported no significant difference between migrant and local patients with TB regarding patient delay time $(16,19)$. One possible explanation for this discrepancy is that migrants in Shanghai had better healthcare access and resources than in other cities (16). Similar to the study by Long et al. (18), our study found that migrant patients with TB had a higher proportion of patients with patient delay $>14$ days. The barriers for migrant access to TB diagnosis included lack of awareness, lack of family and social support, lack of time, and inconvenience of medical insurance reimbursement $(9-11,18)$. It was noteworthy that our study reported shorter patient delays for migrant patients with TB as compared with local patients with TB in 2019. The significantly improved patient delay among migrant patients with $\mathrm{TB}$ is possibly due to the active case detection strategy implemented 
by the local CDC. In 2019, nearly half of the migrant patients with TB were detected from close contact tracing, while in other years they were mainly detected from symptomatic visits. Our study highlights the importance of promoting health education and improving health awareness, especially among migrants to reduce patient delay.

Our study reported a median health system delay of 9 days and a proportion of $42 \%$ with health system delay $>14$ days for migrant patients with TB. This finding is consistent with the results from previous studies, which reported a median health system delay ranging from 8 to 11 days and a proportion ranging from 27 to $45 \%$ with health system delay $>14$ days for migrant patients with TB in China (13, $15,16,19,23)$. Similar to a previous study (23), we found that migrant patients with TB had longer health system delays and a higher proportion of patients with health system delays $>14$ days. One possible explanation for this is that migrant patients with $\mathrm{TB}$ tended to present light symptoms of $\mathrm{TB}$ which could be neglected by some health providers on their first visit to health facilities. Therefore, they need to make several visits to health facilities before being classified as having presumptive TB and referred to a TB designated hospital for TB diagnosis confirmation. Furthermore, some migrants might choose to seek health care back in their hometown when they are classified as presumptive TB. Our study indicates the need to strengthen TB diagnostic training and education for health providers, especially those working at prefectural and higher levels, when consulting migrants with suspected TB symptoms, to provide timely screening or referral to the $\mathrm{TB}$ designated hospitals.

Very few studies have employed survival analysis into the research of delay in TB diagnosis among patients with $\mathrm{TB}$. The study of Chen et al. seemed to be the first study to analyze the diagnostic delay of patients with TB using survival analysis in China (21). In this study, we employed survival analysis to study the probability of being diagnosed at different time intervals for migrant patients with TB as compared with local patients with TB. Our study found that the longer the time interval, the more likely migrant patients with $\mathrm{TB}$ are to be diagnosed as compared with local patients with $\mathrm{TB}$. This again confirms that more serious delays in diagnosis occurred in the migrant patients with TB than the local residents.

\section{Limitations}

Our study has several limitations. First, this study was only conducted in only one county of China, hence the generalizability of our findings is limited. However, with a larger sample size including all registered TB cases from 2015 to 2019, our study provides solid data on the diagnostic delay for migrant patients with TB as compared with local patients with TB. Second, the time of onset of TB symptoms and first visit to a health facility was based on the self-reported information of patients with TB during clinical consultations, thus recall biases might exist. Third, the diagnosis of TB is sometimes difficult due to the confusion between TB and other diseases like lung cancer. In this case, the delay may be prolonged, in addition to the delay caused by the diagnostic process itself as it takes 2 days for three samples of smear sputum to be tested before TB confirmation. In other words, our analysis of health systems delay may dilute the diagnostic process when patients arrive at the TB clinic. Future studies should make differences between provider delay (from the first contact of the health facilities to the visit of the TB clinic) and confirmation delay (from the first visit of the TB clinic to the confirmation of TB). Finally, our study discloses the patterns of differences in diagnostic delays and time points between migrant and local patients for 5 years. Previous studies have suggested that factors such as socio-demographic and clinical characteristics could also influence the patient and health systems delay $(21,35-$ 37). Further studies could be conducted to identify the impact of these confounding factors on the differences in the diagnostic delays between these two groups of patients.

\section{CONCLUSION}

Diagnosis is significantly delayed among migrant patients with TB. Our study highlights the importance of early screening and diagnosis for TB especially among migrants, to improve access and ensure better management for all patients with TB.

\section{DATA AVAILABILITY STATEMENT}

The data analyzed in this study is subject to the following licenses/restrictions: Information in our database is confidential. Requests to access these datasets should be directed to Guanyang Zou, zgy1021@hotmail.com.

\section{ETHICS STATEMENT}

The studies involving human participants were reviewed and approved by Zhejiang Provincial Center for Disease Control and Prevention. Written informed consent for participation was not provided by the participants' legal guardians/next of kin because: This is a patient record review study with data exported from China's Tuberculosis Information Management System. Data recorded in the system and used for this study are collected during TB registration and consultation.

\section{AUTHOR CONTRIBUTIONS}

GZ, WX, and LZ conceived and designed the study. BC and DH participated in data collection and analysis. GZ and WX were major contributors in writing the manuscript. $\mathrm{OC}$ and XW provided constructive suggestions on the study. All authors contributed to the article and approved the submitted version.

\section{FUNDING}

This study was supported by the National Social Science Foundation of China (Grant Number 20\&ZD122) and the 
Zhejiang Provincial Science and Public Welfare Project (LGF19H260004). The funding source was not involved in the design of the study and the collection, analysis and interpretation of data, and writing the manuscript.

\section{REFERENCES}

1. World Health Organization. Global Tuberculosis Report. Geneva (2019). Available online at: https://apps.who.int/iris/bitstream/handle/10665/336069/ 9789240013131-eng.pdf (accessed August 1, 2021).

2. National Bureau of Statistics. Statistical Communiqué of the People's Republic of China on the 2019 National Economic and Social Development (in Chinese). Beijing (2019). Available online at: http://www.stats.gov.cn/tjsj/zxfb/202002/ t20200228_1728913.html (accessed August 2, 2021).

3. Mou J, Griffiths SM, Fong H, Dawes MG. Health of China's rural-urban migrants and their families: a review of literature from 2000 to 2012. Brit Med Bull. (2013) 106:19-43. doi: 10.1093/bmb/ldt016

4. Zeng J, Shi L, Zou X, Chen W, Ling L. Rural-to-urban migrants' experiences with primary care under different types of medical institutions in $<$ city $>$ Guangzhou $</$ city $>$, China. PLoS ONE. (2015) 10:e0140922. doi: 10.1371/journal.pone.0140922

5. Li T, Du X, Shewade HD, Soe KT, Zhang H. What happens to migrant tuberculosis patients who are transferred out using a web-based system in China? PLoS ONE. (2018) 13:e0206580. doi: 10.1371/journal.pone.0206580

6. World Health Organization Regional Office for the Western Pacific. Tuberculosis Control in Migrant Populations: Guiding Principles and Proposed Actions. Manila: WHO Press (2016).

7. Dhavan P, Dias HM, Creswell J, Weil D. An overview of tuberculosis and migration. Int J Tuberc Lung Dis. (2017) 21:610-23. doi: 10.5588/ijtld.16.0917

8. Zou G, Zeng Z, Chen W, Ling L. Self-reported illnesses and service utilisation among migrants working in small-to medium sized enterprises in Guangdong, China. Public health. (2015) 129:970-8. doi: 10.1016/j.puhe.2015.04.015

9. Song X, Zou G, Chen W, Han S, Zou X, Ling L. Health service utilisation of rural-to-urban migrants in Guangzhou, China: does employment status matter? Trop Med Int Health. (2017) 22:82-91. doi: 10.1111/tmi.12801

10. Dang Y, Zou G, Peng B, Ling L. Health service seeking behavior among migrant workers in small and medium-sized enterprises in Guangdong, China: does family migration matter? Bio Med Res Int. (2018) 2018:3620436. doi: 10.1155/2018/3620436

11. Yu C, Lou C, Cheng Y, Cui Y, Lian Q, Wang Z, et al. Young internal migrants' major health issues and health seeking barriers in Shanghai, China: a qualitative study. BMC Public Health. (2019) 19:336. doi: 10.1186/s12889-019-6661-0

12. World Health Organization. Implementing the End TB Strategy: The Essentials. WHO/HTM/TB/2015.31. Geneva (2015). Available online at: https://www. who.int/publications/i/item/implementing-the-end-tb-strategy (accessed August 1, 2021).

13. Zhou C, Tobe RG, Chu J, Gen H, Wang X, Xu L. Detection delay of pulmonary tuberculosis patients among migrants in China: a cross-sectional study. Int $J$ Tuberc Lung Dis. (2012) 16:1630-6. doi: 10.5588/ijtld.12.0227

14. Zhong J, Yin J, Zou G, Hu Y, Walley J, Wang X, et al. Experience of implementing the integrated TB model in Zhejiang, China: a retrospective observational study. Trans $R$ Soc Trop Med Hyg. (2016) 110:24651. doi: $10.1093 /$ trstmh/trw001

15. Wang W, Jiang Q, Abdullah ASM, Xu B. Barriers in accessing to tuberculosis care among non-residents in Shanghai: a descriptive study of delays in diagnosis. Eur J Public Health. (2007) 17:419-23. doi: 10.1093/eurpub/ckm029

16. Lu L, Jiang Q, Hong J, Jin X, Gao Q, Bang H, et al. Catastrophic costs of tuberculosis care in a population with internal migrants in China. BMC Health Serv Res. (2020) 20:832. doi: 10.1186/s12913-020-05686-5

17. Huang Y, Huang J, Su X, Chen L, Guo J, Chen W, et al. Analysis of the economic burden of diagnosis and treatment on patients with tuberculosis in Bao'an district of Shenzhen City, China. PLoS ONE. (2020) 15:e0237865. doi: 10.1371/journal.pone.0237865

18. Long Q, Li Y, Wang Y, Yue Y, Tang C, Tang S, et al. Barriers to accessing $\mathrm{TB}$ diagnosis for rural-to-urban migrants with chronic cough in

\section{ACKNOWLEDGMENTS}

We would like to thank Dr. Keven Bermudez for his help in editing and improving the English language of this manuscript.

Chongqing, China: a mixed methods study. BMC Health Serv Res. (2008) 8:202. doi: 10.1186/1472-6963-8-202

19. Zhao F, Zhu J-F, Tang W-Q, Wang Y, Xu L-X, Chen J-G. The epidemic trend and characteristics of tuberculosis for local population and migrants from 2010 to 2017 in Jiading, China. J Public Health. (2019) 28:33945. doi: 10.1007/s10389-019-01035-x

20. Tobe RG, Xu L, Zhou C, Yuan Q, Geng H, Wang X. Factors affecting patient delay of diagnosis and completion of Direct Observation Therapy, Shortcourse (DOTS) among the migrant population in Shandong, China. Biosci Trends. (2013) 7:122-8. doi: 10.5582/bst.2013.v7.3.122

21. Chen H, Wang T, Liu L, Wang D, Cheng Q. Trend in risk of delay in diagnosis of new pulmonary tuberculosis in Northwest China from 2008 to 2017. BMC Infect Dis. (2019) 19:100. doi: 10.1186/s12879-019$3725-9$

22. Zhejiang Provincial Bureau of Statistics. Zhejiang Statistical Yearbook (in Chinese). Hangzhou (2020). Available online at: http://tjj.zj.gov.cn/col/ col1525563/index.html (accessed August 2, 2021).

23. Xiao W, Huang D, Li S, Zhou S, Wei X, Chen B, et al. Delayed diagnosis of tuberculosis in patients with diabetes mellitus comorbidity and its associated factors in Zhejiang Province, China. $B M C$ Infect Dis. (2021) 21:272. doi: 10.1186/s12879-021-05 929-8

24. Wang Q, Ma A, Han X, Zhao S, Cai J, Kok FJ, et al. Hyperglycemia is associated with increased risk of patient delay in pulmonary tuberculosis in rural areas. $J$ Diabetes. (2017) 9:648-55. doi: 10.1111/1753-0407.12459

25. Li Y, Ehiri J, Tang S, Li D, Bian Y, Lin H, et al. Factors associated with patient, and diagnostic delays in Chinese TB patients: a systematic review and meta-analysis. BMC Med. (2013) 11:156. doi: 10.1186/1741-7015-11-156

26. TB Control Office in Ministry of Health in China. Implementation Manual of the World Bank Tuberculosis Control Program in China. 2nd ed. Beijing: Ministry of Health (1992).

27. Department of Disease Control, Ministry of Health, China; Department of Medical Administration, Ministry of Health, China; Chinese Center for Disease Control and Prevention. Guidelines for implementing the National Tuberculosis Control Program in China (version 2008, in Chinese). Beijing (2018).

28. Ali JS, McDermott S, Gravel RG. Recent research on immigrant health from statistics Canada's population surveys. Can J Public Health. (2004) 95:I913. doi: 10.1007/BF03403659

29. Achkar JM, Sherpa T, Cohen HW, Holzman RS. Differences in clinical presentation among persons with pulmonary tuberculosis: a comparison of documented and undocumented foreign-born versus US-born persons. Clin Infect Dis. (2008) 47:1277-83. doi: 10.1086/592572

30. Mor ZHK, Lidji M, Migliori GB, Leventhal A. Tuberculosis diagnostic delay and therapy outcomes of non-national migrants in Tel Aviv, 1998-2008. Euro Surveill. (2013) 18:pii=20433. doi: 10.2807/ese.18.12.20433-en

31. National Health and Family Planning Commission of China. China Health Statistical Yearbook 2015. Beijing: Peking Union Medical College Press (2016).

32. Liu CH, Li L, Chen Z, Wang Q, Hu YL, Zhu B, et al. Characteristics and treatment outcomes of patients with MDR and XDR tuberculosis in a TB referral hospital in Beijing: a 13-year experience. PLoS ONE. (2011) 6:e19399. doi: 10.1371/journal.pone.0019399

33. Tobe RG, Xu L, Song P, Huang Y. The rural-to-urban migrant population in China: gloomy prospects for tuberculosis control. Biosci Trends. (2011) 5:226-30. doi: 10.5582/bst.2011.v5.6.226

34. Li X, Jiang S, Li X, Mei J, Zhong Q, Xu W, et al. Predictors on delay of initial health-seeking in new pulmonary tuberculosis cases among migrants population in East China. PLoS ONE. (2012) 7:e31995. doi: 10.1371/journal.pone.0031995

35. Lin Y, Enarson DA, Chiang CY, Rusen ID, Qiu LX, Kan XH, et al. Patient delay in the diagnosis and treatment of tuberculosis in China: 
findings of case detection projects. Public Health Action. (2015) 5:659. doi: $10.5588 /$ pha. 14.0066

36. Cai J, Wang X, Ma A, Wang Q, Han X, Li Y. Factors associated with patient and provider delays for tuberculosis diagnosis and treatment in Asia: a systematic review and meta-analysis. PLoS ONE. (2015) 10:e0120088. doi: 10.1371/journal.pone.0120088

37. Sreeramareddy CT, Qin ZZ, Satyanarayana S, Subbaraman R, Pai M. Delays in diagnosis and treatment of pulmonary tuberculosis in India: a systematic review. Int J Tuberc Lung Dis. (2014) 18:255-66. doi: 10.5588/ijtld.13. 0585

Conflict of Interest: The authors declare that the research was conducted in the absence of any commercial or financial relationships that could be construed as a potential conflict of interest.
Publisher's Note: All claims expressed in this article are solely those of the authors and do not necessarily represent those of their affiliated organizations, or those of the publisher, the editors and the reviewers. Any product that may be evaluated in this article, or claim that may be made by its manufacturer, is not guaranteed or endorsed by the publisher.

Copyright (c) 2021 Xiao, Chen, Huang, Chan, Wei, Zhou and Zou. This is an openaccess article distributed under the terms of the Creative Commons Attribution License (CC BY). The use, distribution or reproduction in other forums is permitted, provided the original author(s) and the copyright owner(s) are credited and that the original publication in this journal is cited, in accordance with accepted academic practice. No use, distribution or reproduction is permitted which does not comply with these terms. 